\title{
Retrospective comparative study between 3T WB-MRI including WB-DWI and 18F-FDG-PET/CT in detection of metastatic disease
}

\author{
Yoshikazu Tanaka ${ }^{1 *}$, Katsuyuki Nakanishi ${ }^{1}$, Tadashi Ueda ${ }^{1}$, Tetsuro Nakazawa ${ }^{1}$ and Kazuya Oshima ${ }^{2}$ \\ ${ }^{1}$ Department of Diagnostic and Interventional Radiology, Osaka International Cancer Institute, 3-1-69, Otemae Chuo-ku, Osaka city, Osaka 541-8567, Japan \\ ${ }^{2}$ Department of Orthopaedic Surgery, Osaka International Cancer Institute, 3-1-69, Otemae Chuo-ku, Osaka city, Osaka 541-8567, Japan
}

\begin{abstract}
Purpose: To retrospectively compare the diagnostic accuracy of 18F-FDG-PET/CT (PET-CT) and WB-MRI with WB-DWI (WB-MRI) in various types of metastatic diseases.

Materials and methods: There were 26 patients who underwent WB-MRI and PET-CT within a period of one month. The WB-MRI protocols were: sagittal T1WI (Turbo Spin Echo) with TR/TE: 600/ 9.4 (msec), STIR with TR/ TE: 6000/85/240 (msec), coronal T1WI (FLASH) with TR/TE of 120/2.46 (msec) and axial DWI (b-0 and 800) based on STIR with TR/TE/TI of 14000/60/240 (msec). Three radiologists with various lengths of experience were assigned to review the WB-MRI images. The PET-CT reports were reviewed by radiologists with more than 10 years of experience in diagnostic PET-CT images. All patients had a follow-up of more than 6 months by MRI, computed tomography (CT) or PET-CT.

Results: A total of 124 gold standard malignant lesions were identified. Regarding WB-MRI, the sensitivity was $68-78 \%$ ( $\mathrm{p}=0.059-1.00$ ) and the positive predicted value was 76-84\%. With PET-CT, 98 lesions were detected correctly in total and the sensitivity and positive predictive values were $79 \%$ and $65 \%$. Concerning only skeletal metastasis, the number of gold standard lesions was 80 in total. Regarding WB-MRI, the sensitivity was $85-93 \%$ (p=0.002-0.02) and the positive predicted value was $79-95 \%$. With PET-CT, 61 lesions were detected correctly in total and the sensitivity and positive predictive values were 76\% (61/80), 75\% (61/81) respectively

Conclusion: Although PET-CT and WB-MRI yielded similar overall results in detection of malignant lesions, the performance of WB-MRI in detection of bone metastasis was significantly higher.
\end{abstract}

\section{Introduction}

In oncology patients, accurate whole body assessment is crucial in terms of diagnosis, treatment plan and follow-up. Positron emission tomography (PET)/computed tomography (CT) with 2-(fluorine18)-fluro-2-deoxy-D-glucose (18F-FDG) has become a widely used imaging modality for whole body oncology assessment. 18F-FDG is a mimic of glucose and accumulates in malignant tumors where glucose metabolism is active. Introduction of fused PET-CT scanner bestowed anatomical information of the CT scanner to the 18F-FDG PET in a single examination, thus improving its diagnostic accuracy $[1,2]$.

Magnetic resonance imaging(MRI) is a widely available clinical imaging technique, which has the advantage of providing excellent soft tissue contrast and high spatial resolution without using ionizing radiation. For malignancies involving bone marrow, T1 imageweighted spin-echo (SE) sequences used together with fat-suppressed short tau inversion recovery (STIR) has been described as the most accurate [3-6]. Diffusion weighted image (DWI) has been utilized in evaluation of malignant tumors as cell density is generally high [7]. Although the clinical use of MRI for the whole body had been limited because of its long scanning time and poor image quality, technological advances in the last decade have solved these dilemmas rendering MRI as a feasible candidate for whole body malignancy scan.
The purpose of this study is to verify the diagnostic accuracy of 3T WB-MRI with WB-DWI including WB-DWI (hereafter WB-MRI) and 18 FDG-PET/CT (hereafter PET-CT) in metastatic disease.

\section{Materials and methods}

\section{Patients}

From January 2013 to January 2014, approximately 320 patients with histologically proven malignancies underwent WB-MRI. Of these patients, twenty-six (male, female 39 80 years old, mean age 63.6) had also undergone 18FDG-PET/CT within a period of one month. The number of individual types of neoplasms was: nine cases of lung cancer, seven cases of breast cancer, three cases of head and neck cancer, two cases of esophageal cancer, one case each of gastric cancer, prostate cancer, ovarian cancer, fallopian tube cancer, and soft tissue mucinous liposarcoma. All patients gave informed consent for undergoing both

Correspondence to: Yoshikazu Tanaka, Department of Diagnostic and Interventional Radiology, Osaka International Cancer Institute, 3-1-69, Otemae Chuo-ku, Osaka city, Osaka 541-8567, Japan, E-mail: yoscit@gmail.com

Key words: WB-MRI, 18 FDG-PET/CT, oncology, bone metastasis

Received: September 02, 2017; Accepted: September 22, 2017; Published: September 25, 2017 
modalities. This retrospective study was approved by our institutional review board (IRB).

\section{Magnetic resonance imaging}

A 3 tesla MRI (Magnetom Trio 3T, Siemens, Erlangen, Germany) was utilized. The patients were covered with a 102 channel surface coil, positioned head first on an extended anatomical coverage table.

Sagittal images of STIR and T1WI (Turbo Spin Echo), coronal images of T1WI (FLASH), and axial images of DWI from lower neck to proximal femur were captured. Imaging parameters were: The Sagittal images were acquired by two stations. T1WI (Turbo Spin Echo): TR (Repetition time)/TE (the echo time) was 600/9.4 millisecond (msec). The slice thickness was $4 \mathrm{~mm}$ and the acquisition time (TA) for each station was 1 minute 11 seconds. STIR: TR/TE/TI (Inversion time) was $6000 / 85 / 240 \mathrm{msec}$. The slice thickness was $4 \mathrm{~mm}$, and the TA for each station was 1 minute and 48 seconds.

Coronal T1WI (FLASH) was acquired by two or three stations. The TR/TE was $120 / 2.46 \mathrm{msec}$. The slice thickness was $4 \mathrm{~mm}$ and the TA for each station was 34 seconds ( 2 breath-holds).

DWI (b-0 and 800 ) based on STIR was obtained by four or five stations. The TR/TE/TI was $14000 / 60 / 240 \mathrm{msec}$. The imaging time for each station was 2 minutes 39 seconds. The b- 800 axial images were reformatted on a stack-by-stack basis as a single $4 \mathrm{~mm}$-thick maximum intensity projection image (MIP) in coronal plane and $4 \mathrm{~mm}$-thick multiplanar reformations (MPR) in coronal plane. Thereupon, fused images between the coronal T1 images and coronal DWI (MIP) were created.

The time required by the patients to be immobile in the MRI bore was approximately 30 minutes. The total examination time, including the coil set up, patient positioning, image processing (MIP, MPR and Fusion) and the image layout for reading, was less than 60 minutes.

\section{(18)F-FDG-PET-CT}

For FDG-PET/CT, either Biograph Duo (Siemens, Erlangen, Germany) with two lines of detectors or Gemini (Philips Medical Systems, Best, the Netherlands) with 16 lines of detectors was used. After a fasting period of a minimum of 6 hours, blood glucose was measured ensuring it was less than $140 \mathrm{mg} / \mathrm{dl}$. 18F-FDG was then administered in a standard dose of $3.7 \mathrm{MBq} / \mathrm{Kg}, 60$ minutes before the scan. The patients were instructed to void immediately prior to the scan. PET and CT scans were obtained from vertex to femur under free breathing.

\section{Analysis and counting}

Malignant lesions were recorded on an illustrated predefined sheet. We counted the lesions according to the modified classification proposed by Lausenstein, et al. [8] The skeletal system was divided into 13 regions, which were skull, cervical spine, thoracic spine, lumbar spine, sacrum, coccyx, sternum, rib, clavicle, scapula, humerus, ilium, femur. Lymph nodes were counted in regions, which were axillary, cervical, thoracic abdominal and inguinal regions. Other metastases sites included the lungs, pleura, liver, adrenal glands, peritoneum,etc. In cases where there were more than two metastases in the same region or organ, they were counted as two. If there were bilateral lesions in bilateral organs or bones, such as the lungs or scapula, these were counted as two.

Three radiologists with various lengths of experience were assigned to review the WB-MRI images. The only information provided to the readers was that the WB-MRI had been taken for malignancy follow-up. They were allowed to examine the given WB-MRI images only. Centricity PACS ${ }^{\mathrm{R}}$ Radiology RA1000 was used for the image assessment.

The number of lesions which the readers assessed as malignant were counted. These were typically lesions appearing as hypo-intense areas on T1WI, hyper-intense areas in STIR and DWI.

For 18 FDG-PET/CT, the radiological reports made by specialist radiologists in diagnostic 18FDG-PET/CT imaging were retrospectively examined and the lesions which were clearly indicated as malignant were counted.

\section{Definition of malignant lesions (gold standard)}

All the cases had a follow-up of at least six months by computed tomography (CT), MRI, PET-CT or plain roentgenogram. The gold standards were set on agreement of a radiologist and a physician who were not directly involved in conducting the study.

The lesions were defined as true positives if they were pathologically proven to be a metastatic lesion or revealed a typical malignant course in the follow-up imaging studies. In cases with ongoing chemotherapy or radiotherapy, the lesions showing diminution or typical changes in response to the treatment were counted as true positives.

The lesions considered as malignant by WB-MRI or PET-CT but later pathologically proven to be non-metastatic or showed no change or diminution in the follow-up imaging studies were counted as false positives.

The lesions considered malignant by WB-MRI or PET-CT but later pathologically proven to be metastatic or demonstrated increase in size in the follow-up imaging studies were counted as true positives.

Newly appeared lesions after the initial evaluation by WB-MRI or PET-CT were not counted in the final number of malignant lesions.

\section{Statistics}

Assessing the true positives and false positives was determined according to the defined gold standard and sensitivity and positive predictive values for both modalities were calculated. For WB-MRI, each reader's sensitivity and positive predictive values were calculated. The McNemer test was applied to obtain the diagnostic agreement of both modalities with the gold standard. Statistical significance was considered in cases with p-values less than 0.05 .

Cohen's Kappa was calculated to assess the WB-MRI interobserver agreement. Kappa values of greater than 0.61 were considered significant in interobserver agreement.

\section{Results}

The number of gold standard lesions was 124 in total. WB-MRI interobserver agreement was acceptable with kappa values of 0.70 between readers 1 and 2, 0.66 between 2 and 3, and 0.66 between 1 and 3. Regarding WB-MRI, the sensitivity was $68-78 \%(\mathrm{p}=0.0059-1.00)$ and the positive predicted value was $76-84 \%$. With FDG-PET 98 lesions were detected correctly in total and the sensitivity and the positive predictive value were $79 \%$ and $65 \%$ (Table 1).

A representative case of PET-CT demonstrating superior metastatic lymph node detection is shown in Figure 1.

Concerning only the skeletal metastasis, the number of gold standard lesions was 80 in total. Regarding WB-MRI, the sensitivity 
A

B

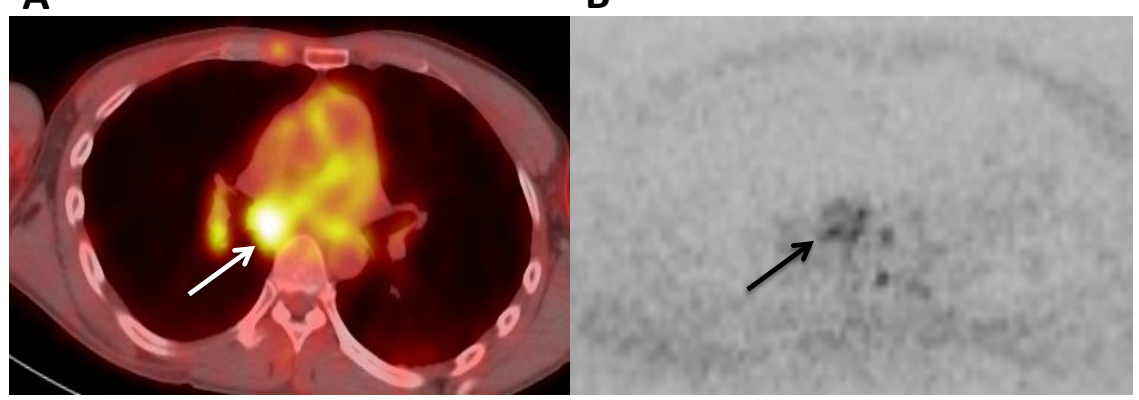

C

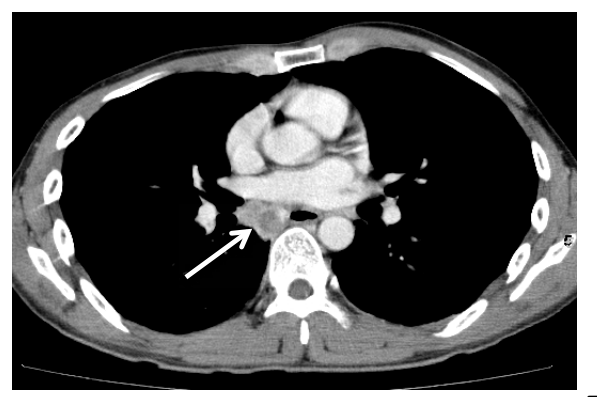

D

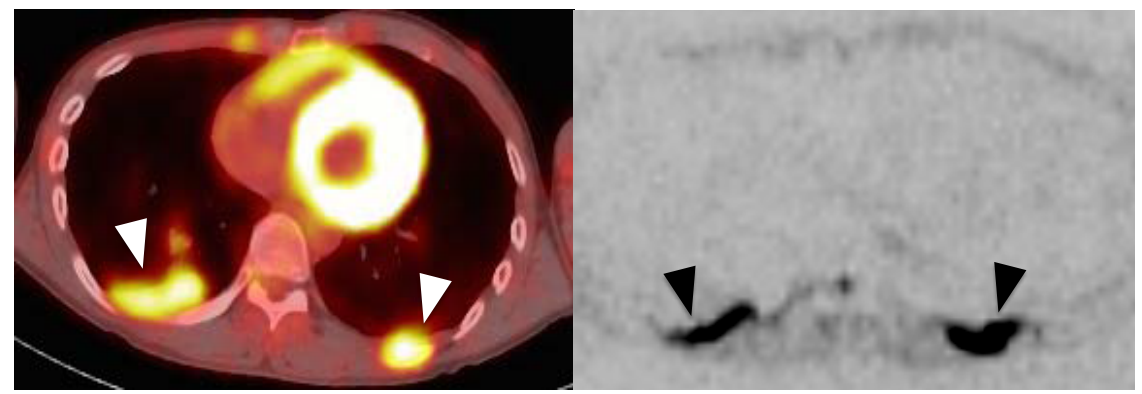

Figure 1. 43-year-old male with non-small cell lung cancer.

A. Axial image of FDG-PET shows accumulation in mediastinum (white arrow) and was diagnosed as lymph node metastasis.

B. In the axial image of DWI at the same level as Fig.1A, although the lymph node is appearing slightly hyperintense (arrow), no readers could point out the abnormality.

C. Follow-up contrast enhanced CT image shows an enlarged lymph node and was verified as a gold standard lesion (white arrow).

D. Axial image of DWI slightly caudal to Fig.1B reveals high signal in the pleural disseminations (arrowheads). Note their obvious signal intensity compared to the Fig.B lymph node signal. All readers pointed out these lesions.

Table 1. Summary of overall metastasis results

\begin{tabular}{|c|c|c|c|}
\hline \multicolumn{2}{|c|}{} & Sensitivity & $\begin{array}{c}\text { Positive Predictive } \\
\text { Value }\end{array}$ \\
\hline \multirow{3}{*}{ WB-MRI } & Reader1 & $68 \%(84 / 124), \mathrm{p}=0.059$ & $76 \%(84 / 111)$ \\
\cline { 2 - 4 } & Reader2 & $78 \%(97 / 124), \mathrm{p}=1.00$ & $84 \%(97 / 115)$ \\
\cline { 2 - 4 } & Reader3 & $75 \%(93 / 124), \mathrm{p}=0.52$ & $76 \%(93 / 123)$ \\
\hline & 18 FDG-PET/CT & $79 \%(98 / 124)$ & $65 \%(98 / 136)$ \\
\hline
\end{tabular}

was $85-93 \%(\mathrm{p}=0.002-0.21)$ and the positive predicted value was 79 95\%. With FDG-PET, 61 lesions were detected correctly in total and the sensitivity and the positive predictive value were $76 \%$ and $75 \%$ (Table 2 ).

A representative case of superior sensitivity of WB-MRI detecting bone lesions earlier than 18FDG-PET/CT is demonstrated in Figure 2.

\section{Discussion}

Although 18FDG-PET/CT has been the choice of modality for assessment of metastatic disease, technological advances in the last decade have solved some technological dilemmas realizing MRI as a feasible candidate for whole body malignancy scan.
Table 2. Summary of skeletal metastasis results

\begin{tabular}{|c|c|c|c|}
\hline \multicolumn{2}{|c|}{} & Sensitivity & $\begin{array}{c}\text { Positive Predictive } \\
\text { Value }\end{array}$ \\
\hline \multirow{3}{*}{ WB-MRI } & Reader1 & $85 \%(68 / 80), \mathrm{p}=0.21$ & $89 \%(68 / 76)$ \\
\cline { 2 - 4 } & Reader2 & $92 \%(72 / 80), \mathrm{p}=0.045$ & $95 \%(72 / 76)$ \\
\cline { 2 - 4 } & Reader3 & $93 \%(74 / 80), \mathrm{p}=0.002$ & $79 \%(74 / 94)$ \\
\hline & 18 FDG-PET/CT & $76 \%(61 / 80)$ & $75 \%(61 / 81)$ \\
\hline
\end{tabular}

An important technology involved in shortening the examination time is parallel imaging (PAT). With this technique, independent radiofrequency receiver coils covering the whole body permit data acquisition from a multitude of body parts to obtain a reconstructed whole body image without compromising spatial or temporal resolution.

Another remarkable technique in WB-MRI is the image acquisition under normal breathing with multiple signal averaging, invented by Takahara, et al. [9] This new method has improved SNR, enabling thin slice DWI image acquisition, which can be reconstructed as whole body DWI 3D MIP images similar to PET. 
A

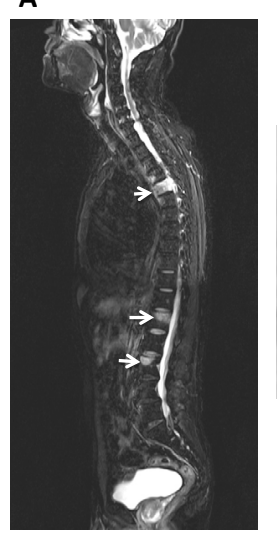

D

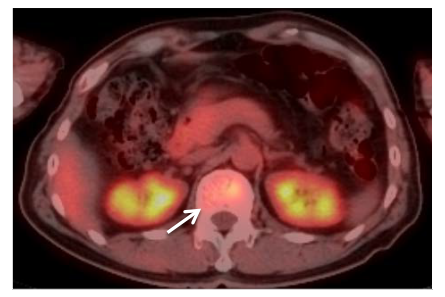

$\mathbf{F}$

B
C
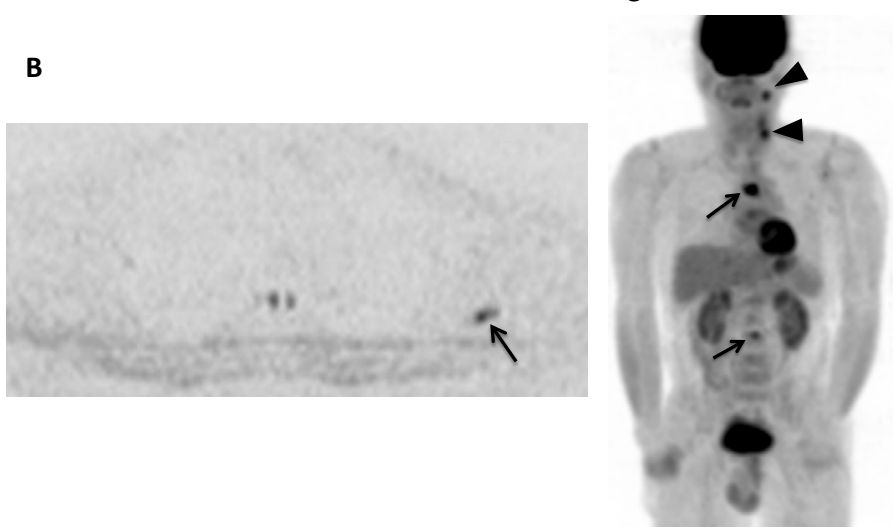

$\mathbf{E}$

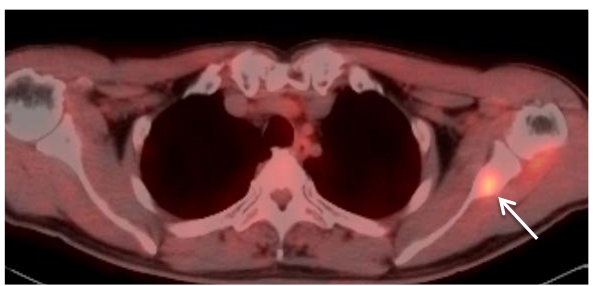

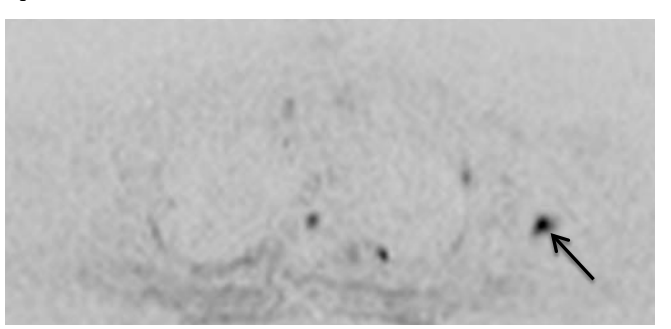

Figure 2. 61-year-old male with hypopharyngeal cancer.

A. Total spine sagittal STIR image shows high intensity areas in Th5, L1, L3 and were diagnosed as bone metastases (white arrows).

B. Axial DWI shows high intensity area in the left scapula (arrow). Two of three readers pointed out the lesion.

C. MIP image of FDG-PET shows FDG avid areas in Th5 and L3 (arrows) but not in L1 and the left scapula. There is also FDG accumulation in the left cervical lymph nodes (arrowheads).

D. Approximately 90 days after first FDG-PET/CT examination, a follow-up FDG-PET/CT was taken. A FDG avid area had appeared in L1 (white arrow).

E. In the same examination as in Fig.2D, an FDG avid area in left scapula had appeared (white arrow).

F. Approximately 150 days after the first WB-DWI examination, WB-MRI was repeated. The high intensity area of the left scapula had become more obvious (arrow).

Both the left scapula lesion and the L1 lesion were verified as gold standards.

Both the left scapula lesion and the L1 lesion were verified as goldstandard metastases.

Our overall sensitivities for detection of malignant lesions were similar in both modalities and the positive predictive value of PETCT was lower than that of WB-MRI. The WB-MRI yielded superior sensitivity and positive predictive values in detection of bone metastases. 18FDG-PET/CT counted more false positive skeletal lesions, which resulted in a lower overall positive predictive value.

Anotch et al conducted TNM staging in various oncologic diseases with both modalities [10]. The results showed overall sensitivity was similar for the detection of distant metastasis, however, for assessment of bone metastasis, the sensitivity of WB-MRI was significantly higher. In our study, which included WB-DWI, yet, this observation was similar.

Yang et al. examined 56 patients with mixed tumors, demonstrating the important role of DWI WB-MRI in tumor detection [11]. Among the cases, 12 patients underwent also FDG-PET. DWI WB-MRI showed the highest sensitivity and specificity in bone metastasis. Diagnostic superiority of WB-MRI in bone metastasis was also observed in our study.
Regarding bone metastasis, a meta-analysis of comparative studies of WB-DWI, bone scintigraphy and 18 FDG-PET/CT, has already been published and it concluded that WB-MRI was more accurate and cost effective [12]. Among other studies, Schmidt et al. prospectively compared the diagnostic accuracy of WB-MRI and 18FDG-PET/CT in different oncologic diseases and WB-MRI demonstrated a sensitivity, specificity and accuracy of $94 \%, 76 \%$ and $91 \%$ [13]. Although our study did no yield specificity and accuracy, the result is rather similar. Moreover, Heusner et al investigated the diagnostic ability of bone metastasis in non-small cell lung cancer and melanoma and concluded FDG-PET-CT and WB-MRI were equally effective [14].

Confronting results have been reported by Cafagna et al. who conducted a retrospective study in detection of metastasis including different types of tumors and concluded that 18 FDG-PET/CT remained a better choice in identifying bone metastases [15].

Daldrup-Link et al reported discordant results comparing WBMRI with FDG-PET and bone scintigraphy in children and young 
adults. FDG-PET showed the highest sensitivity among the modalities resulting: WB-MRI $82 \%$, FDG-PET $90 \%$ and bone scintigraphy $71 \%$ [16]. With the younger age group, this result could be speculated by the higher cellularity of the bone marrow appearing as low signal intensity on T1-weighted images and a hyperintense signal on fat-suppressed sequences, reducing the contrast of malignant lesions in the bone marrow.

In our study, significant numbers of false positive lesions were counted with PET-CT. On a lesion by lesion analysis, possible degenerative changes in vertebral endplates were mistaken for metastasis in 11 lesions. Since FDG is a non-tumor specific tracer, it also accumulates in sites with inflammatory changes.

Since FDG-PET/CT was taken in $5 \mathrm{~mm}$ slice thickness without sagittal or coronal images in this study, there could have been an advantage in observation of lesions juxtapositional to the vertebral end plates for WB-MRI with sagittal STIR and T1WI images. We also noted an advantage of WB-MRI in a case of mucinous liposarcoma where multiple bone lesions were imperceptible on PET-CT [17].

WB-MRI may have disadvantages in detecting metastatic lesions in the thoracic cage where motion artifacts of respiration and pulsation impair image quality $[3,16,18]$. In this study with added WB-DWI, there were 10 rib metastases and all WB-MRI readers detected all rib lesions. It can be speculated that addition of WB-DWI improves the peripheral bone metastasis diagnoses. Also, WB-DWI images fused with coronal T1 images may have further facilitated detection of peripheral skeletal lesions. It has been reported that MRI has greater sensitivity and permits earlier visualization of bone lesions than conventional bone scintigraphy [19-22]. We have encountered a comparable case where a bone metastasis appeared first in WB-MRI and was later detected by the follow-up 18FDG/PET-CT. More high-quality prospective studies are awaited to investigate this finding.

There are several limiting aspects in this study. Firstly, the gold standards were based on observational radiological images without histological diagnostic proves. In this situation, slow growing lesions and lesions without morphological changes may not have been included in the gold standard. This could have increased the false negative counts. Thirdly, the number of cases was limited to 26 , making it susceptible to random errors. Lastly, the counting of 18 FDG-PET/ CT lesions was purely based on a retrospective manner based on radiological reports.

In conclusion, although PET-CT and WB-MRI yielded similar overall results in detection of malignant lesions, the performance of WB-MRI in detection of bone metastasis was significantly higher.

\section{Acknowledgment}

Authors acknowledge the MRI technical staff for cooperating in the WB-MRI image acquisition and processing.

\section{References}

1. Even-Sapir E, Metser U, Flusser G, Zuriel L, Kollender Y, et al. (2004) Assessment of malignant skeletal disease: initial experience with $18 \mathrm{~F}$-fluoride PET/CT and comparison between 18F-fluoride PET and 18F-fluoride PET/CT. J Nucl Med 45: 272278. [Crossref]

2. Pelosi E, Messa C, Sironi S, Picchio M, Landoni C, et al. (2004) Value of integrated $\mathrm{PET} / \mathrm{CT}$ for lesion localisation in cancer patients: a comparative study. Eur J Nucl Med Mol Imaging 31: 932-939. [Crossref]

3. Walker R, Kessar P, Blanchard R, Dimasi M, Harper K, et al. (2000) Turbo STIR magnetic resonance imaging as a whole-body screening tool for metastases in patients with breast carcinoma: preliminary clinical experience. J Magn Reson Imaging 11: 343350. [Crossref]
4. Mehta RC, Marks MP, Hinks RS, Glover GH, Enzmann DR (1995) MR evaluation of vertebral metastases: T1-weighted, short-inversion-time inversion recovery, fast spin-echo, and inversion-recovery fast spin-echo sequences. Am J Neuroradiol 16: 281-288. [Crossref]

5. Kellenberger CJ, Miller SF, Khan M, Gilday DL, Weitzman S, et al. (2004) Initial experience with FSE STIR whole-body MR imaging for staging lymphoma in children. Eur Radiol 14: 1829-1841. [Crossref]

6. Mahnken AH, Wildberger JE, Adam G, Stanzel S, Schmitz-Rode T, et al. (2005) Is there a need for contrast-enhanced T1-weighted MRI of the spine after inconspicuous short tau inversion recovery imaging? Eur Radiol 15: 1387-1392. [Crossref]

7. Guo Y, Cai YQ, Cai ZL, Gao YG, An NY, et al. (2002) Differentiation of clinically benign and malignant breast lesions using diffusion-weighted imaging. J Magn Reson Imaging 16: 172-178. [Crossref]

8. Lauenstein TC, Freudenberg LS, Goehde SC, Ruehm SG, Goyen M, et al. (2002) Whole-body MRI using a rolling table platform for the detection of bone metastases. Eur Radiol 12: 2091-2099. [Crossref]

9. Takahara T, Imai Y, Yamashita T, Yasuda S, Nasu S, et al. (2004) Diffusion weighted whole body imaging with background body signal suppression (DWIBS): technica improvement using free breathing, STIR and high resolution 3D display. Radiat Med 22: 275-282. [Crossref]

10. Antoch G, Vogt FM, Freudenberg LS, Nazaradeh F, Goehde SC, et al. (2003) Whole-body dual-modality PET/CT and whole-body MRI for tumor staging in oncology. JAMA 290: 3199-3206. [Crossref]

11. Yang TH, Lin JZ, Wang X, Lu JH, Chen Z (2008) Preliminary study of feasibility of whole body diffusion weighted imaging in diagnosis of metastasis of tumor. Chin Med Sci J 23: 187-192. [Crossref]

12. Wu LM, Gu HY, Zheng J, Xu X, Lin LH, et al. (2011) Diagnostic value of whole-body magnetic resonance imaging for bone metastases: a systemic review and meta-analysis. J Magn Reson Imaging 34: 128-135. [Crossref]

13. Schmidt GP, Schoenberg SO, Schmid R, Stahl R, Tiling R, et al. (2007) Screening for bone metastases: whole-body MRI using a 32-channel system versus dual-modality PET-CT. Eur Radiol 17: 939-949. [Crossref]

14. Heusner T, Gölitz P, Hamami M, Eberhardt W, Esser S, et al. (2011) "One-stopshop" staging: should we prefer FDG-PET/CT or MRI for the detection of bone metastases? Eur J Radiol 78: 430-435. [Crossref]

15. Cafagna D, Rubini G, Iuele F, Maggialetti N, Notaristefano A, et al. (2012) Whole-body MR-DWIBS vs. [18F]-FDG-PET/CT in the study of malignant tumors: a retrospective study. Radiol Med 117: 293-311. [Crossref]

16. Daldrup-Link HE, Franzius C, Link TM, Laukamp D, Sciuk J, et al. (2001) Wholebody MR imaging for detection of bone metastases in children and young adults: comparison with skeletal scintigaphy and FDG PET. AJR Am J Roentgenol 177: 229236. [Crossref]

17. Berger KL, Nicholson SA, Dehdashti F, Siegel BA (2000) FDG PET evaluation of mucinous neoplasms: correlation of FDG uptake with histopathologic features. AJR Am J Roentgenol 174: 1005-1008. [Crossref]

18. Steinborn MM, Heuck AF, Tiling R, Bruegel M, Gauger L, et al. (1999) Whole-body bone marrow MRI in patients with metastatic disease to the skeletal system. $J$ Comput Assist Tomogr 23: 123-129. [Crossref]

19. Nakanishi K, Kobayashi M, Nakaguchi K, Kyakuno M, Hashimoto N, et al. (2007) Whole-body MRI for detecting bone tumor: diagnostic value of diffusion-weighted images. Magn Reson Med Sci 6: 147-155. [Crossref]

20. Hargaden G, O'Connell M, Kavanagh E, Powell T, Ward R, et al. (2003) Curren concepts in whole-body imaging using turbo short tau inversion recovery MR imaging. AJR Am J Roentgenol 180: 247-252. [Crossref]

21. Engelhard K, Hollenbach HP, Wohlfart K, von Imhoff E, Fellner FA (2004) Comparison of whole-body MRI with automatic moving table technique and bone scintigraphy for screening for bone metastases in patients with breast cancer. Eur Radiol 14: 99-105. [Crossref]

22. Gutzeit A, Doert A, Froehlich JM, Eckhardt BP, Melli A, et al. (2010) Comparison of diffusion-weighted whole body MRI and skeletal scintigraphy for the detection of bone metastases in patients with prostate or breast carcinoma. Skeletal Radiol 39: 333-343. [Crossref]

Copyright: (C2017 Tanaka Y. This is an open-access article distributed under the terms of the Creative Commons Attribution License, which permits unrestricted use, distribution, and reproduction in any medium, provided the original author and source are credited. 